\title{
Prácticas de visualización en la investigación académica en diseño gráfico
}

\section{Resumen}

Esta investigación explora las prácticas académicas alrededor de la visualización, con el objetivo de definir la importancia de la realización de esquemas para la investigación académica en diseño. El estudio de caso se centró en los estudiantes de últimos semestres de Diseño Gráfico de la Universidad del Valle que estaban realizando su proyecto de grado para optar al título universitario. Métodos: en esta investigación cualitativa se realizó observación participativa de las asignaturas de proyectos de grado, además de entrevistas semiestructuradas a profesores del departamento y a estudiantes próximos a finalizar sus estudios profesionales. En la parte final de la entrevista, a los estudiantes se les pidió que explicaran sus proyectos mientras graficaban sobre papel. Se llevo a cabo un análisis de la relación entre los gráficos y la sustentación verbal; en el estudio de las entrevistas transcritas se realizó un escrutunio dentro de la conversación para identificar las categorías de visualización, escritura académica e investigación en diseño. Resultados: La información recogida muestra que la visualización es una práctica comunicativa fundamental en el desarrollo de los proyectos de grado, no solo para dialogar con pares y asesores, sino también como una manera de hacer conscientes los procesos cognitivos que hacen parte del proceso investigativo. Conclusión: La incorporación efectiva de métodos visuales en las clases de investigación formativa podría ayudar a los estudiantes a utilizar con mayor eficacia recursos cognitivos adquiridos en sus años de formación, e inclusive colaborar a reducir la brecha percibida entre el ejercicio profesional del diseño y la reflexión teórica sobre la práctica. Futuros estudios podrían centrarse en la aplicación de estos recursos didácticos en las actividades de investigación para comprobar si es posible definir una caja de herramientas metodológicas afín a los intereses particulares del diseño.

\author{
María Fernanda Ramírez \\ Escobar \\ Profesora auxiliar, Departamento de \\ Diseño, Universidad del Valle \\ Cali, Colombia. \\ Correo electrónico: \\ maria.f.ramirez@correounivalle.edu.co \\ ๑ orcid.org/0000-0002-3559-0309 \\ Google Scholar
}

Recibido: julio 27 de 2018 Aprobado: junio 08 de 2020

Palabras clave:

visualización, prácticas académicas, pensamiento visual, investigación en diseño. 


\section{Visualization Practices in Academic Research in Graphic Design}

\begin{abstract}
This research explores academic practices about visualization, with the objective of defining the importance of the realization of diagrams for academic research in design. The case study focused on students in the last semesters of the Bachelor of Arts jn Graphic Design at Universidad del Valle who were carrying out their undergraduate research project to qualify for the university degree. Methods: in this qualitative research, participatory observation of the subject areas dealing with research projects was carried out, in addition to semi-structured interviews with department professors and students about to finish their undergraduate studies. In the final part of the interview students were asked to explain their projects while drawing on a paper. An analysis of the relationship between the graphics and the oral defense of projects was carried out. Scrutiny within the conversation, to identify the categories of visualization, academic writing and design research, was carried out in the analysis of the transcribed interviews. Results: The information collected shows that visualization is a fundamental communicative practice in the development of undergraduate research projects, not only to discuss with pairs and advisors, but also as a way to raise awareness of cognitive processes that are part of the research process. Conclusion: The effective incorporation of visual methods in training research classes could help students use more effectively cognitive resources acquired in their training years at the university and even to reduce the perceived gap between the professional practice of design and the theoretical reflection about the practice. Future studies could focus on the application of these didactic resources in research activities to check if it is possible to define a methodological toolbox related to the particular interests of design.
\end{abstract}

Key words:

visualization, academic practices, visual thinking, design research. 
En los programas de pregrado en Diseño que se ofrecen en Colombia es común el requisito de presentar como proyecto de grado un documento que debe dar cuenta de un proceso de investigación que por lo general desemboca en la realización de un proyecto. Por supuesto, se concibe este tipo de investigación, también llamada investigación formativa, como diferente de la que se realiza en los niveles de formación avanzada. En otros países se reserva el término investigación para escenarios de posgrado, aunque se reconoce la importancia del desarrollo de habilidades básicas de investigación en los estudiantes de pregrado, que puedan ser aplicadas al desarrollo del proyecto de diseño (Restrepo, 2008).

En el Departamento de Diseño de la Universidad del Valle se han categorizado tres tipos de investigación formativa para el desarrollo del proyecto de grado. La primera, proyectos de diseño o investigación y desarrollo (también conocida por estudiantes y profesores como proyecto de intervención), transita "en torno a la relación entre sujeto, contexto y necesidad, entendidas como variables de una realidad socialmente construida" (Universidad del Valle, 2017). Este tipo de proyectos coincide en sus lineamientos generales con la investigación para el proyecto que usualmente es realizada en los programas de diseño, donde la finalidad es el proyecto mismo (Restrepo, 2008). El segundo tipo de proyecto de grado es el proyecto teórico de diseño (conocido también en el departamento de diseño como proyecto de análisis). En este caso la investigación se centra "en asuntos conceptuales y teóricos del Diseño Gráfico o del Diseño industrial que permiten una reflexión rigurosa sobre la profesión y alguna de las distintas líneas de aplicación; busca plantear problemas de orden teórico y/o práctico" (Universidad del Valle, 2017). Este tipo de proyecto de grado, al ser de corte teórico, no desemboca en una propuesta práctica de diseño. Por último, se contempla como opción de proyecto de grado el proyecto de creación en diseño. En este caso, se trata de una propuesta de orden creativo, "en la cual el estudiante estructura un desarrollo de tipo exploratorio sobre el diseño, 
obteniendo una aplicación que da como resultado, además de la aplicación, una propuesta teórico-metodológica del proceso desarrollado" (Universidad del Valle, 2017).

Algunos estudiantes presentan grandes dificultades para realizar el proceso de investigación que es requisito para optar por el título universitario. Particularmente, la formalización del documento escrito que da cuenta del proceso es percibida como un requisito desvinculado de las habilidades desarrolladas en los años de estudio. Los problemas con la escritura académica no son exclusivos del diseño: en la universidad, por lo general, aprender a escribir es una labor que se delega a unos pocos cursos de escritura que no están integrados al saber específico de cada disciplina, es decir, la escritura no se asume como una actividad incluida de forma esencial al currículo (Carlino, 2004). En el caso del diseño, la distancia con la escritura se acrecienta al haber un énfasis en el desarrollo de otro tipo de habilidades distintas a la conceptualización teórica.

En la enseñanza del diseño, intervienen otras competencias diferentes a las de áreas del conocimiento como son las ciencias sociales o las ciencias exactas. La representación, la visualización de alternativas a un problema dado y la elaboración de bocetos, maquetas o modelos son importantes para desarrollar habilidades clave en la práctica profesional. Aunque la lectura y la reflexión hacen parte de la disciplina, el énfasis principal de la carrera no es la construcción teórica por medio de la escritura. El programa de pregrado en Diseño de la Universidad del Valle no es la excepción; aunque se encuentran materias teóricas de análisis y documentación, el eje de la carrera son los talleres de diseño, donde es fundamental el desarrollo de otro tipo de recursos cognitivos diferentes a la conceptualización teórica (como la solución creativa de problemas y la materialización de propuestas). Al llegar a los últimos semestres, el estudiante se enfrenta a una dinámica completamente diferente. 
Asignaturas como metodología de investigación y proyectos de grado exigen del estudiante otro tipo de competencias distintas a las habilidades desarrolladas en torno al desarrollo de proyectos, en los talleres de diseño.

A partir de la observación de los procesos de investigación formativa entre los estudiantes, y con el conocimiento de la importancia didáctica de los diagramas y esquemas conceptuales en la educación, surgió una hipótesis de trabajo. Si los esquemas utilizan tanto palabras, como imágenes y símbolos para representar información, su lenguaje híbrido podría ser una buena manera de acercar a los estudiantes de diseño - ya acostumbrados a pensar gráficamente a través de sus años de estudio- al rigor metodológico de la investigación académica. Para abrir el camino a la discusión, y antes de explicar el método empleado, se hará una sinopsis de algunos conceptos sobre visualización, investigación en diseño y prácticas de visualización en la educación en diseño.

\section{Fundamentos teóricos}

\section{Los esquemas como herramienta para la comprensión}

La acción de representar por medio de gráficos y esquemas el conocimiento está ligada al hombre, a la manera en que percibe espacialmente su entorno y comprende su realidad inmediata. El mapa, como representación simbólica del espacio real, es tal vez la evidencia más antigua de la necesidad del hombre de registrar su comprensión del espacio para compartir su conocimiento con otros, o, en otras palabras, comunicar su memoria espacial. En palabras de Harley:

Como mediadores entre el mundo psíquico interior y el mundo exterior físico, los mapas son herramientas fundamentales para ayudar a la mente humana a encontrar sentido a su universo en varias escalas. Son indudablemente, una de las formas más antiguas de comunicación humana. (Harley, 1987, p. 1). 


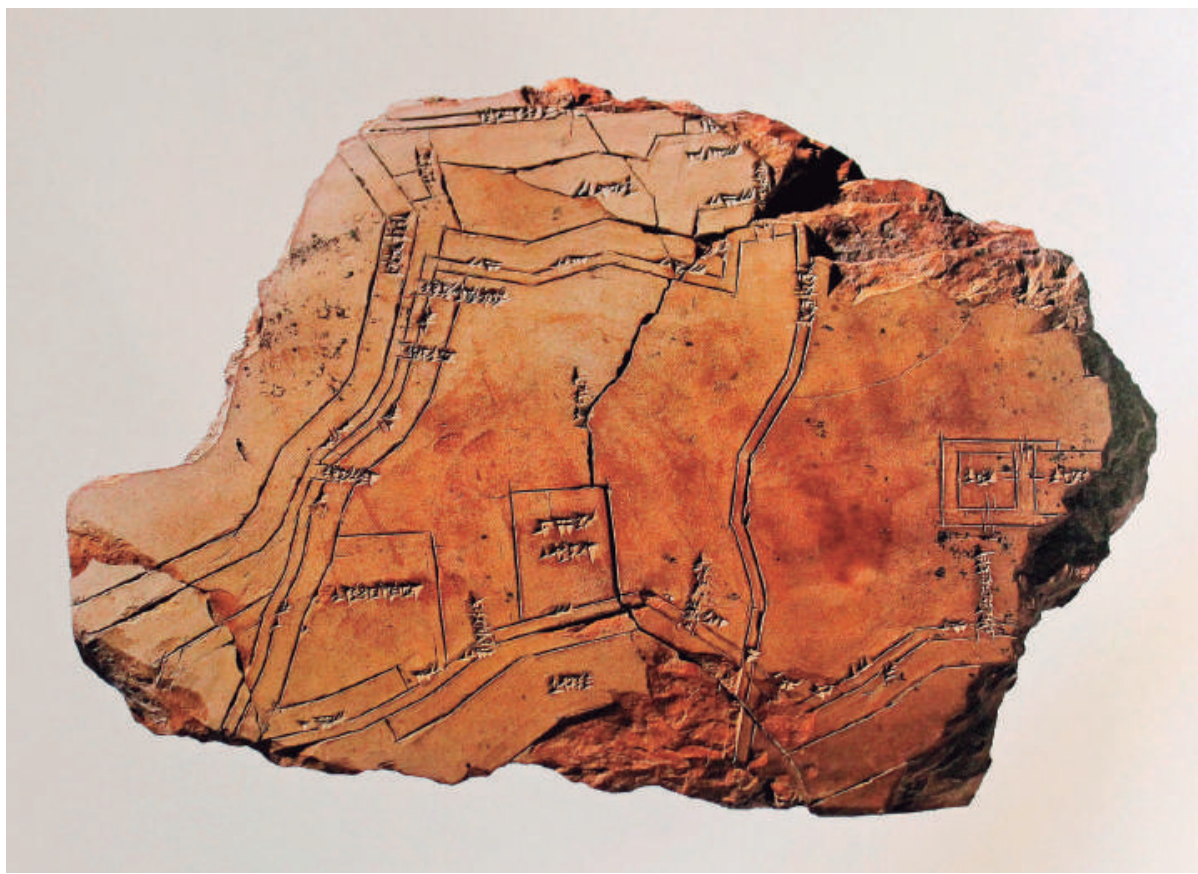

Imagen 1. Mapa de Nippur (1400 a. C.) Nota: Un ejemplo en la antigüedad de la realización de esquemas son los mapas de arcilla de la Antigua Babilonia, de los cuales el mapa de Nippur (1400 a. c.) es tal vez uno de los ejemplos más conocidos. Fuente: León, J. M. (2018). El plano de la ciudad [Fotografía]. https://laciudadvisitada.blogspot.com/2018/04/el-plano-de-la-ciudad.html

Si el mapa es en un inicio un registro del mundo visible, la capacidad humana de ubicar espacialmente situaciones o conceptos abstractos permite que los mapas hayan sido empleados durante siglos como metáforas y herramientas útiles para el pensamiento. Para explicar con mayor precisión esta idea, Tversky (2005) establece que: "tal vez por la ubicuidad del razonamiento espacial, por la naturalidad con que elaboramos mapas de elementos abstractos y los relacionamos con objetos en el espacio, el razonamiento espacial sirve como 
una base para el conocimiento abstracto y la inferencia" (p. 209). Así como la cartografía se vincula desde la antigüedad a la relación del hombre con el espacio percibido o imaginado, las matemáticas, y en particular la geometría, han utilizado desde sus comienzos los esquemas como un medio para comunicar las ideas, y también como una herramienta para pensar sobre estas ideas (Diezmann, 2009).

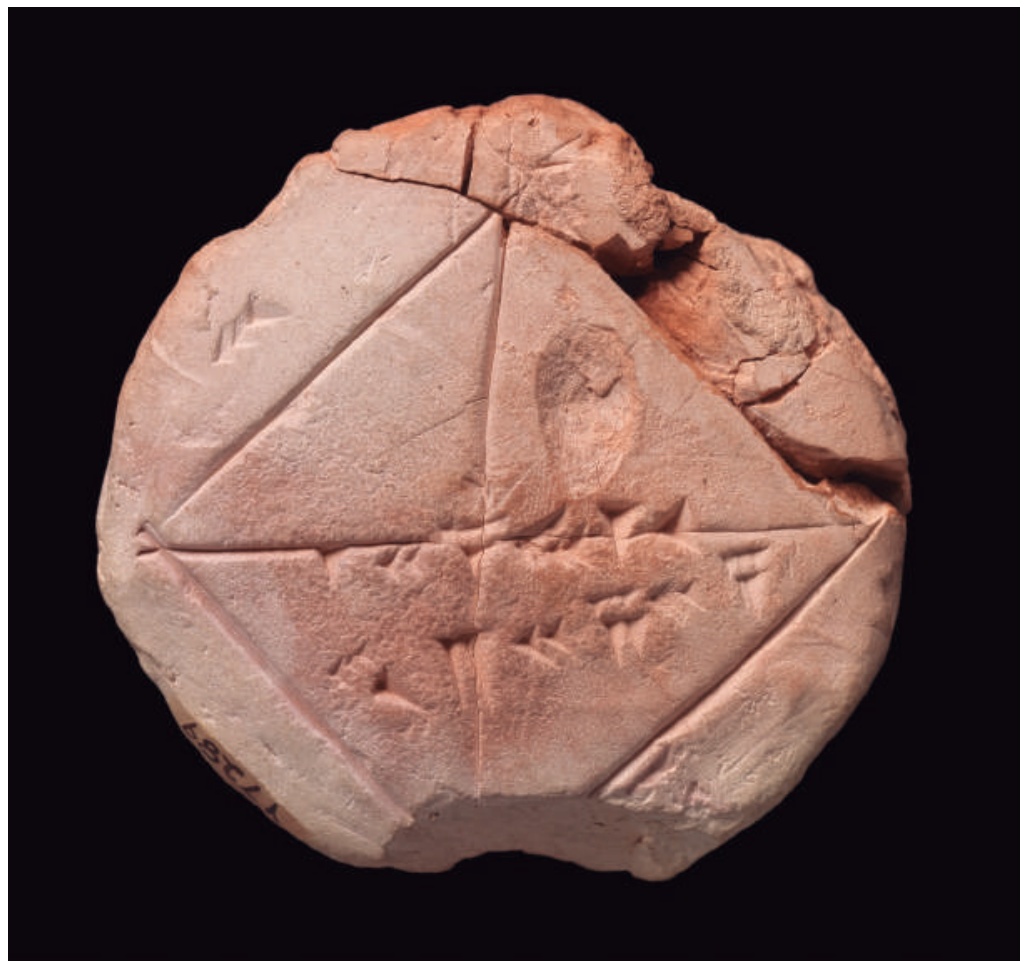

Imagen 2. Fotografía de una tableta de arcilla que muestra una aproximación de la raíz cuadrada del número 2 . Fuente: Casselman, B. (2018). YBC 7289 [Fotografía]. http://www.math.ubc.ca/ cass/Euclid/ybc/ybc.html 
Al revisar ejemplos históricos se puede concluir que en el inicio de la representación espacial de la información, la cartografía, la astronomía y la geometría, constituyen diferentes maneras de acercarse a la representación del espacio conocido o imaginado (Friendly, 2007).

Los esquemas han sido utilizados desde la antigüedad para compartir conocimiento, pero ¿qué hace que sean útiles en el proceso investigativo? Se presentan a continuación tres argumentos a favor del uso de la visualización en procesos investigativos; el primero de ellos es la capacidad de los gráficos de mostrar con claridad patrones emergentes en el análisis de los datos (Rajamanickam, 2005; Hansen, 2000; Tufte, 1992), en segundo lugar, se explora la relación entre la visualización y el pensamiento creativo (Hansen, 2000). Por último, se define el concepto de inteligencia espacial (Gardner, 1993), que permite entender con mayor claridad la utilidad de los mapas mentales para establecer relaciones entre conceptos.

Un ejemplo del uso de los patrones visuales para la inferencia lo constituye el mapa realizado por el Dr. Jhon Snow (ver imagen 3), quien ubicó las muertes de cólera en Londres en un mapa de la ciudad. La epidemia, que tenía perplejos a los investigadores, mostró en el gráfico una relación antes pasada por alto: el cólera se había extendido en los alrededores de una bomba de agua específica. La relación entre la bomba de agua contaminada podría en efecto haberse encontrado de otra manera, a través de cálculos y deducciones; pero es evidente que la visualización permitió leer de manera mucho más eficaz los datos (Tufte, 1992). 


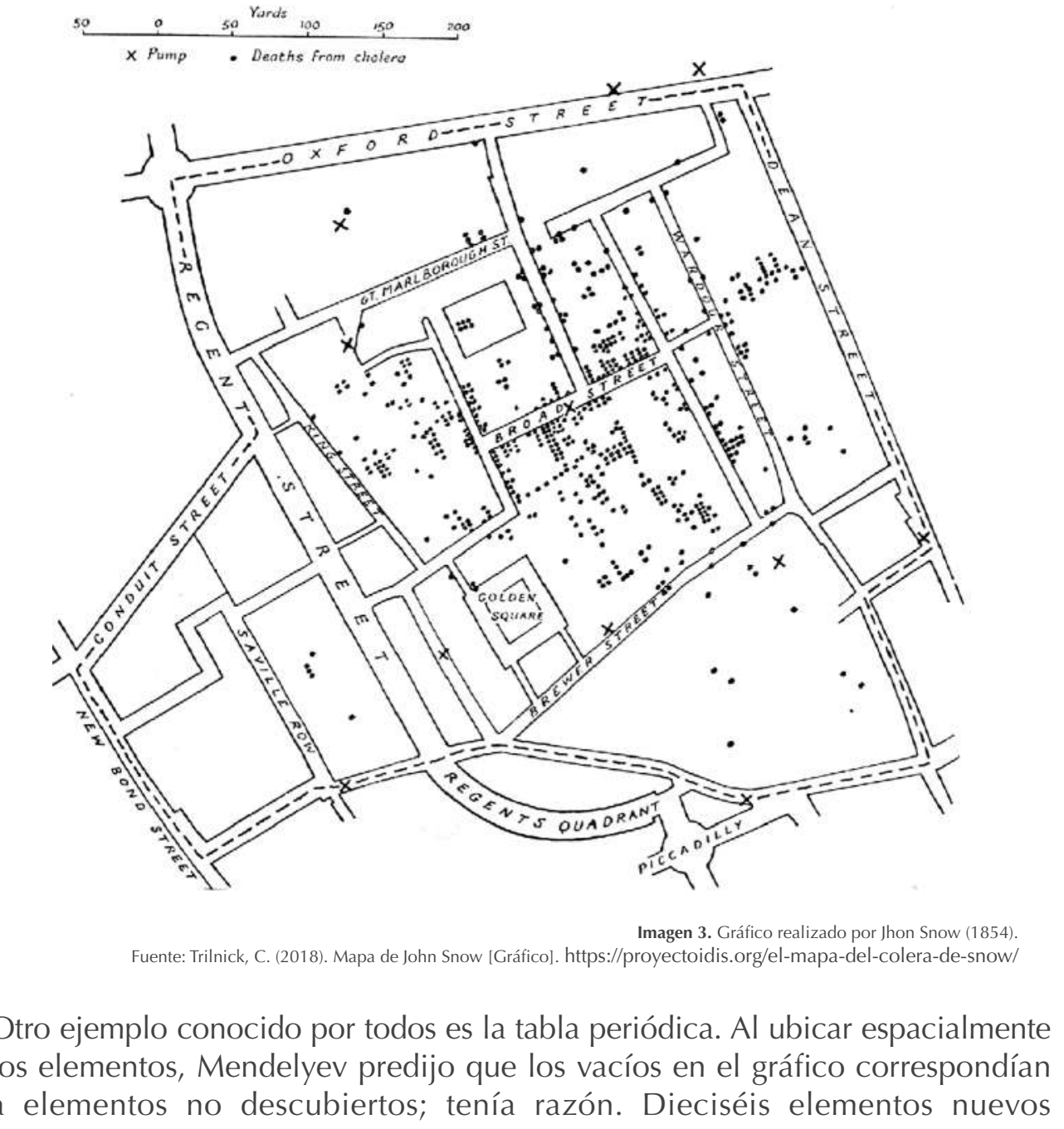


ingresaron a la tabla desde entonces y sus propiedades corresponden a las predichas por el químico. Este ejemplo demuestra cómo la organización de la información permite predecir irregularidades a ser resueltas (Rajamanickam, 2005). Al registrar los datos espacialmente, las repeticiones, el ritmo, e inclusive la ausencia de los elementos en relación, revelan en la superficie del papel la evidencia que parece escapar a la conceptualización más abstracta del lenguaje oral y escrito. Por otro lado, la relación entre síntesis creativa y visualización aparece a menudo en las anécdotas de investigadores. Un sueño revelador, una imagen que debe ser consignada con rapidez en el papel antes que desaparezca, el esquema de una solución todavía desordenada y confusa sobre una servilleta.

La creencia que las ideas y las palabras ocurren simultáneamente en la mente, y el pensamiento no existe mas allá del lenguaje verbal, se contradice en una frase que se escucha a menudo cuando alguien trata de explicar una idea que lucha por acceder a la conciencia y logra evadir las etiquetas y los rótulos: "no puedo encontrar las palabras correctas". Esta afirmación parece indicar que las ideas existen en algún lugar más allá de las palabras; y en realidad, a pesar del énfasis dado en la educación tradicional a la palabra escrita, existen instancias que escapan al lenguaje verbal (Hansen, 2000). Las razones por las que existe una conexión entre la visualización y los procesos creativos no son del todo claras, pero podría tener relación con algunas investigaciones sobre el papel de los hemisferios cerebrales en las funciones cognitivas.

Teniendo en cuenta lo peligrosas que resultan las simplificaciones cuando se trata de hablar de algo tan complejo como el pensamiento, es conocida la relación que hace la neurociencia entre el hemisferio derecho del cerebro y las habilidades espaciales. El hemisferio derecho parece desempeñar un papel en el pensamiento holístico, relacional, intuitivo; mientras el hemisferio 
izquierdo tiene importancia en el uso del lenguaje verbal, en las habilidades matemáticas y en el pensamiento secuencial, lógico, racional (Heilman, 2005). No leemos una imagen en secuencia, entendemos su sentido de manera global. En consecuencia, el pensamiento espacial tiene más relación con la síntesis sincrónica de la etapa de "iluminación" del proceso creativo, que la etapa analítica de comparación de conceptos, más afín a procesos de tipo secuencial. Con la ayuda de imágenes estaríamos entonces motivando lo que Moles y Costa Ilamaban "pensamiento en superficie", en contraposición al pensamiento lineal, propio del lenguaje escrito (Moles y Costa 1991). Finalmente, los esquemas permiten establecer relaciones entre conceptos, al poner en práctica una habilidad muy antigua, que aseguró la supervivencia a nuestros ancestros; la capacidad de orientarse en el espacio.

\section{Inteligencia espacial}

La inteligencia espacial reúne varias habilidades independientes entre sí pero que usualmente trabajan en conjunto. La capacidad de reconocer diferentes instancias del mismo elemento, evocar una escena en la mente y transformarla, reconocer la transformación de un elemento en otro, producir una representación gráfica a partir de la información espacial y otras habilidades relacionadas (Gardner, 1993). La inteligencia espacial no se utiliza únicamente cuando se logra concluir exitosamente el reto de parquear un automóvil en un espacio pequeño, o cerrar una maleta de viaje después de incluir muchas más prendas de lo que podría parecer posible; puede ser entender cómo destruir un tumor sin dañar tejido sano, diseñar un museo o inclusive inferir si una conclusión sigue de forma lógica sus premisas. Todas estas acciones, algunas de índole práctica y otras mucho más abstractas, utilizan el razonamiento espacial (Tversky, 2005). También se emplea este tipo de razonamiento cuando se trabaja con representaciones gráficas — versiones bi o tridimensionales del mundo real—al igual que otros símbolos, como mapas o diagramas. 
El ser humano comienza a aprender sobre su entorno desde el momento de su nacimiento y aún antes de salir del vientre materno; el conocimiento espacial es fundamental para la supervivencia $y$, como toda habilidad esencial, se comienza a desarrollar muy temprano en la vida. Es tan común la inferencia a partir de la comprensión del espacio que el lenguaje está plagado de referencias espaciales. "Tratamos de mantener nuestros espíritus arriba, trabajar en la cúspide de nuestras habilidades, evadir caer en depresiones, zanjas o atolladeros; entramos en terrenos abiertos, luchamos por permanecer en la cima y no perder la profundidad" (Tversky, 2005, p. 14).

Aunque los estudios sobre habilidades espaciales se centraron en principio exclusivamente en la capacidad del ser humano de orientarse en el espacio, pronto fue evidente que la habilidad de establecer relaciones espaciales entre elementos del entorno podía extrapolarse a la relación entre conceptos que permite el aprendizaje. Ausubel, Novak y Henesian, pensadores de tendencia constructivista, afirmaron que aprendemos con mayor facilidad si logramos entender las relaciones entre conceptos, más que la definición de cada concepto como unidad aislada. La relación de los conceptos nuevos con el conocimiento previo se da a través de lo que se denomina "ideas de anclaje". El aprendizaje significativo plantea que se recuerda con mayor facilidad conceptos que "significan" algo para quien aprende, es decir, que están relacionados con otros conceptos previos que presentan particular importancia en los esquemas mentales. El aprendizaje se da en la integración entre el nuevo conocimiento y las estructuras cognitivas existentes, que son esquemas jerárquicos de conceptos Ilamados mapas conceptuales (Ausubel, Novak y Henesian, 1978). Si se considera que en el aprendizaje interactúan las relaciones entre los conceptos a manera de un mapa mental, se puede entender por qué en ocasiones, a través de la representación visual de modelos conceptuales, se pueden entender conceptos de una manera más clara que a partir únicamente de la linealidad del texto. 


\section{Investigación en Diseño}

El diseño posibilita nuevas experiencias en la vida cotidiana de la sociedad, experiencias en el manejo de productos, signos y servicios, incluidas experiencias estéticas que a su vez están sometidas a una dinámica sociocultural. Como la ciencia, el diseño produce cambios en la sociedad, pero a diferencia del científico que busca generar nuevo conocimiento, el diseñador observa el mundo desde la perspectiva de la realización de proyectos y la solución de problemas (Bonsiepe, 2007). Si esta divergencia marca diferencias en la práctica profesional, con mayor razón implica una distancia en las actividades relacionadas con la investigación. Entre profesionales del diseño es común considerar investigación a la recolección de datos que permite tomar mejores decisiones respecto al producto final del proceso proyectual, sin embargo, este tipo de indagación no es considerada como Investigación (con "I" mayúscula), porque no tiene el rigor metodológico que debe estar presente en la investigación académica. "La diferencia radica en que una investigación sólo aporta un nuevo conocimiento para el investigador, pero no para el mundo, mientras que una Investigación realiza un aporte para ambos" (Pontis, 2009). En resumen, no toda acción de investigar lleva a una investigación. El término investigación, en prácticas proyectuales, debería utilizarse solo si dicho proceso aporta conocimiento inédito para la disciplina. Una investigación académica se considera como tal solo si es sistemática, conducida intencionalmente para adquirir nuevos conocimientos, puede ser comprobada y, además, los conocimientos adquiridos sobre un tema pueden ser trasmitidos y dados a conocer (Scrivener, 2009).

Con respecto a la investigación en diseño, Frayling (1993) propone una clasificación que ha ganado popularidad dentro de la comunidad académica. Clasifica los tipos de investigación en investigación para el diseño (research for design), investigación sobre el diseño (research about design) e investigación a 
través del diseño (research through design). Findeli et al. (2008) profundizan en dicho paradigma, que se explica a continuación:

La acción de investigar en aras de obtener información para un proyecto de diseño (lo que anteriormente se mencionaba como investigación con " $\mathrm{i}$ " minúscula), corresponde a lo que se ha denominado investigación para el diseño (research for design). Este tipo de investigación es relevante para la práctica del diseño al asegurarse que todos los parámetros relacionados con el desarrollo del proyecto (ergonómicos, psicológicos, estéticos, etc.) estén bien definidos. No es considerado aceptable como investigación académica, por varias razones. En primer lugar, usualmente no genera nuevo conocimiento, se nutre de información ya disponible. En segundo lugar, aunque se llegue a generar nuevo conocimiento, por lo general el proceso no es realizado con el rigor metodológico esperado por los estándares científicos, lo que hace difícil su comprobación. Por último, en muchos casos la información obtenida debe permanecer confidencial, al ser parte del desarrollo de producto de una empresa específica (Findeli et al, 2008).

La investigación sobre el diseño (research about design) es por lo general realizada por disciplinas diferentes al diseño, de acuerdo con parámetros científicos. El problema radica, en este caso, en que, aunque sean investigaciones avaladas por la comunidad académica, son poco relevantes para la disciplina del diseño. Al ser los investigadores teóricos de otras disciplinas antropólogos, historiadores, semiólogos, etc., no poseen la experiencia necesaria para comprobar que el conocimiento que están produciendo sea útil para la disciplina del diseño (Findeli et al, 2008).

Por último, la investigación a través del diseño (research through design) es entendida como una investigación que posee lo mejor de las dos clasificaciones anteriores. Es entonces un tipo de investigación que, además de ser conducida 
con el rigor metodológico avalado por la academia, produce contenido original sobre el diseño que es pertinente para la disciplina (Findeli et al, 2008).

Por supuesto, para definir qué es investigación en diseño es necesario tener en mente una definición sobre el diseño. Es en este sentido que, en las últimas décadas, se ha evidenciado un cambio importante: de definir el diseño como ciencia a definir el diseño como una disciplina. Con esta diferencia de énfasis el foco está ahora en entender el proceso de diseño a través de la comprensión del proceso cognitivo que acompaña la acción de diseñar, o para decirlo de otra manera, las formas de pensar y conocer que son propias del diseño (Cross, 2007). Si existe una manera de conocer el mundo que es propio del diseño, podríamos asumir también que hay métodos de investigación particulares que corresponden a los intereses de la disciplina. Sin embargo, el diseño emplea frecuentemente metodologías de investigación que pertenecen a las ciencias sociales o inclusive a las ciencias experimentales.

Volviendo a las categorías descrita por Frayling (1993), podríamos afirmar que la investigación sobre el diseño (research about design) corresponde también a algunas investigaciones que, aunque rigurosas y dirigidas por diseñadores, pueden estar desconectadas de la realidad disciplinar, al trasladar de manera literal teorías, lineamientos y metodologías de las ciencias sociales o de otras áreas del conocimiento. El diseño se caracteriza por tener una base teórica poco desarrollada en comparación con las ciencias sociales, por lo que es común apoyarse en otras ramas del conocimiento, como la filosofía, la semiótica o la historia. La ausencia de un cuerpo teórico sólido en diseño puede tener muchos motivos. Uno de ellos puede ser el escaso énfasis durante la formación en las materias de corte teórico. Al ser la enseñanza de las disciplinas proyectuales primordialmente de tipo práctico, la carga horaria de las asignaturas de taller es mucho mayor. Otro factor que ha contribuido a esta situación 
Es la identificación del diseñador como "persona práctica"; aunque no haya nada que indique que la práctica (acción) es lo que sigue a la reflexión (investigación) o que es la reflexión lo que sigue a la práctica, o que una excluya a la otra. (Pontis, 2009, p. 8)

Como contribución a estas maneras de pensar y conocer propias del diseño (Cross, 2007), y buscando definir procesos metodológicos propios de la disciplina, se propone estudiar en mayor profundidad los métodos visuales como parte del proceso de investigación en diseño. Como disciplina proyectual, el diseño se apoya en gran medida en la realización de bocetos, dibujos y maquetas. La representación gráfica para la educación y práctica del diseño se ha estudiado principalmente desde la importancia de los dibujos rápidos o bocetos, un tipo fundamental de representación en las etapas de diseño conceptual (Maya y Mazo, 2020; Self, Lee y Bang, 2015). En cambio, la aplicación de métodos visuales en la investigación académica no se ha estudiado con mayor profundidad, a pesar de la relación estrecha del diseño con la representación como herramienta cognitiva. Esto por supuesto, está cambiando, como lo prueban la serie de talleres Visual Methods Workshop, en universidades del Reino Unido (Pontis, 2009).

Con el propósito de definir si la realización de esquemas puede ser un recurso importante para la formación investigativa de los estudiantes de diseño, la investigación se centró en los estudiantes de últimos semestres de Diseño Gráfico de la Universidad del Valle en proceso de realizar su proyecto de grado para optar al título universitario. En particular, se estudiaron las prácticas académicas alrededor de la visualización.

El concepto de práctica académica puede definirse “(...) como un conjunto de actividades institucional y socialmente reconocidas para cumplir las funciones universitarias de docencia y proyección social. Estas prácticas tienen que ver con los procesos de enseñanza y aprendizaje, la producción intelectual y la difusión del conocimiento" (Rincón y Gil, 2013, p. 35). Estas prácticas dan 
lugar a ciertos tipos de saberes y competencias compartidos. Cada disciplina es un marco dentro del que organiza el conocimiento usando para ello métodos particulares de acercarse a la realidad. En el caso de la comunidad académica del diseño, por las particularidades que definen su matriz disciplinar, se podría incluir la visualización como una de las competencias académicas propias.

\section{Método}

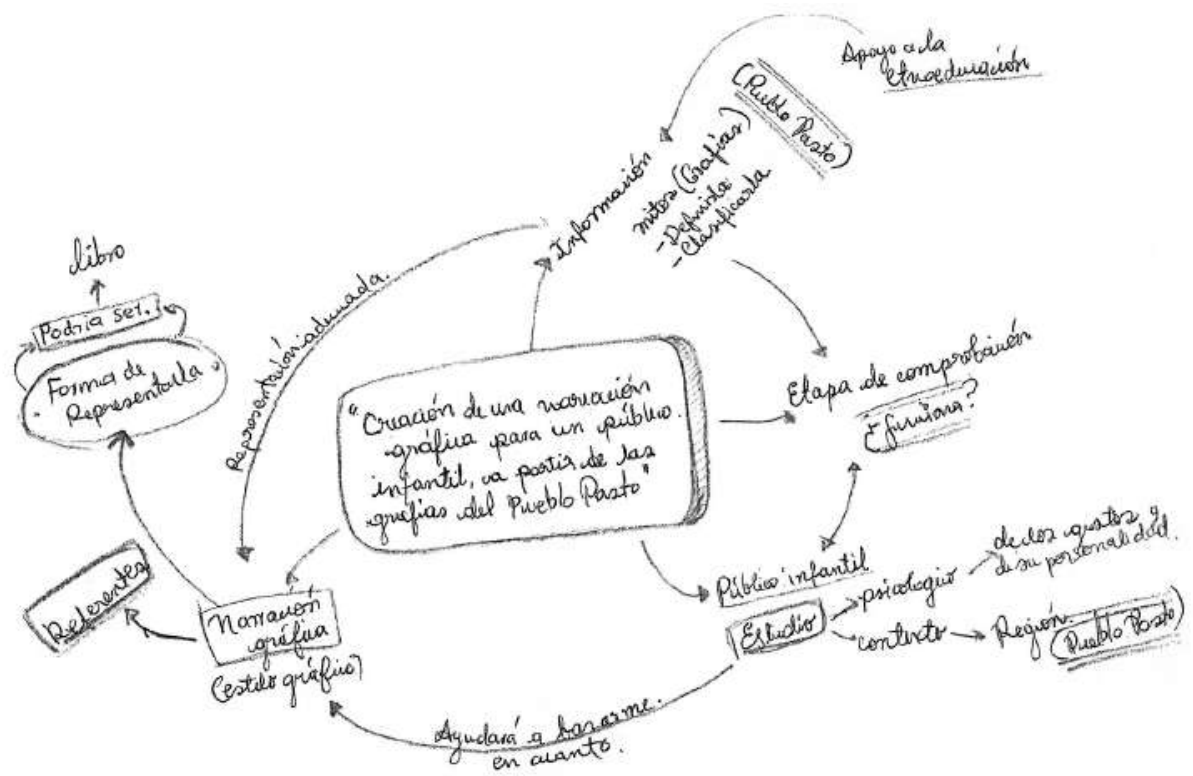

Imagen 4. Gráfico realizado en el marco de las actividades académicas de Proyecto de grado II en Diseño Gráfico. 
En primera instancia, se llevó a cabo una revisión bibliográfica para explorar los conceptos de visualización, la relación entre visualización y comprensión de ideas, y las competencias propias de la investigación en diseño. En la clase de proyectos de grado 1 y 2, se realizó observación participativa desde la asesoría de los proyectos de grado a los estudiantes (ver imagen 4). Como parte de la indagación, se realizaron entrevistas a ocho estudiantes de Diseño Gráfico que se encontraban realizando su trabajo de grado y a tres profesores del Departamento de Diseño de la Universidad del Valle.

En la entrevista a los estudiantes, las preguntas se dirigían a entender el papel que jugó la visualización y la elaboración de bocetos y alternativas de diseño en los primeros semestres de la carrera; también se indagó sobre el papel que cumplía en estos semestres iniciales la escritura académica y la investigación como parte de la formación profesional. Posteriormente se buscó entender la importancia de estos mismos conceptos en los últimos semestres de la carrera, donde las asignaturas de metodología de la investigación y proyecto de grado I y II constituyen el terreno donde se desarrolla el proyecto de grado.

Una vez concluida esta primera parte de la entrevista se les pidió a los participantes que hablaran de sus proyectos de grado en desarrollo con una solicitud concreta: debían explicar sus proyectos de investigación mientras graficaban sobre papel (ver imágenes 5 y 6). Se acordó con tres de los estudiantes realizar un seguimiento posterior, pues la conversación inicial había revelado procesos sobre los que era útil recopilar una mayor información. En estas conversaciones posteriores se recogieron gráficos elaborados por los estudiantes como parte de su proceso personal, bocetos y escritos relacionados con el desarrollo del proyecto de investigación y quedaron registrados los cambios en el discurso a medida que avanzaba el proceso del trabajo de grado. 


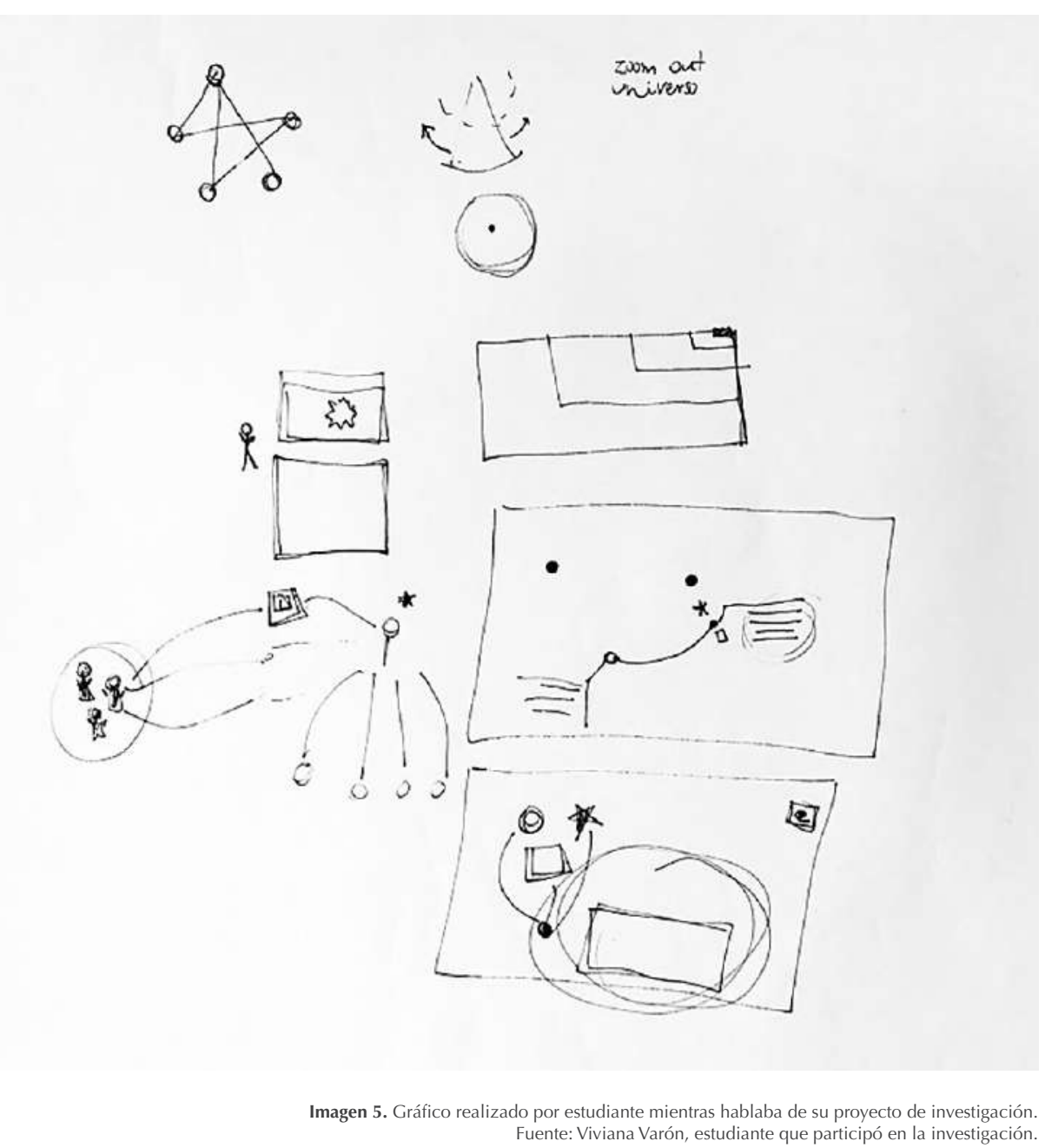




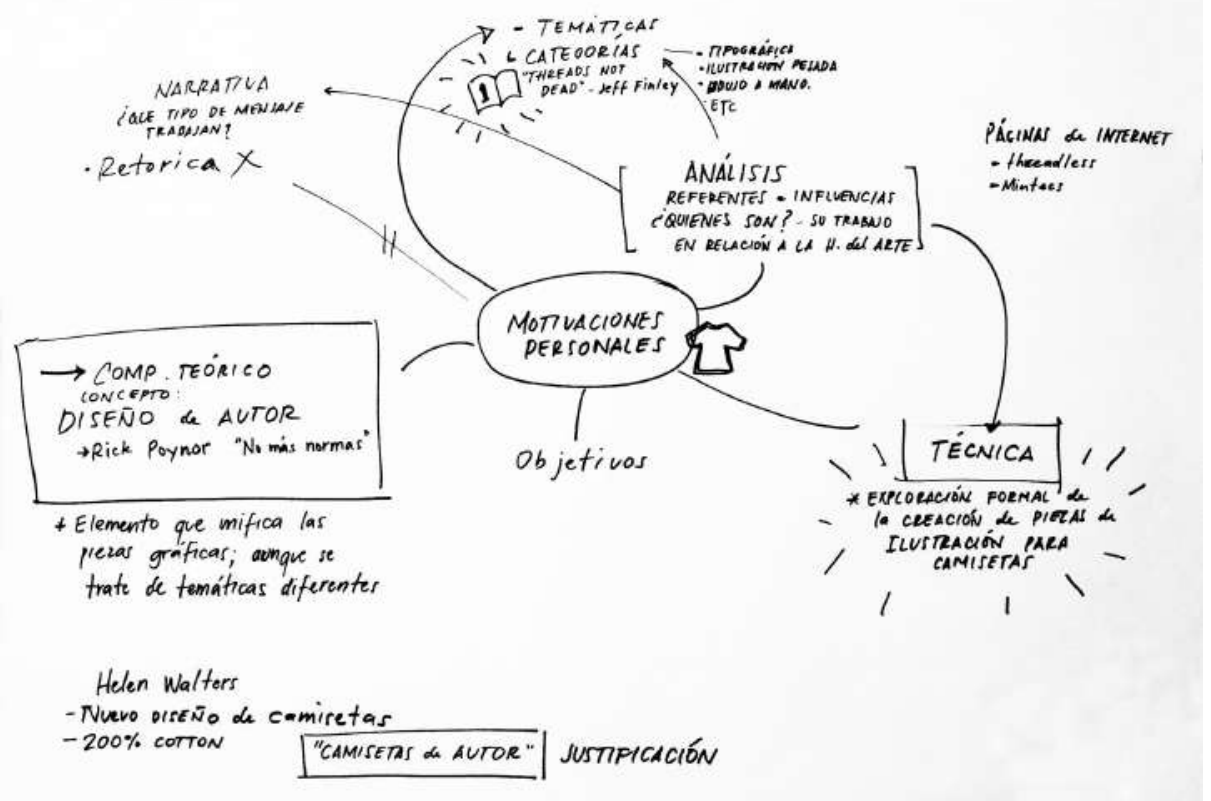

Imagen 6. Gráfico realizado por estudiante mientras hablaba de su proyecto de investigación. Fuente: Miguel Montenegro, estudiante que participó en la investigación.

En la entrevista a los docentes, además de indagar sobre las relaciones entre visualización e investigación en su formación como diseñadores, se exploró cómo aplicaban estas prácticas académicas en sus planes de clase. Los esquemas realizados por los estudiantes durante las entrevistas se analizaron en tres niveles de significación. En el primero, se estudió la imagen en sí misma a partir de sus elementos gráficos constitutivos, el color, la línea, la composición, el grado de abstracción, la jerarquía entre los elementos. En un segundo nivel, se buscaron las relaciones entre la imagen y el discurso verbal, la explicación del proyecto que hizo el estudiante a 
medida que intervenía el papel blanco; y, en un tercer nivel de análisis, se estableció un diálogo entre el gráfico realizado, el discurso verbal y otro tipo de documentos recogidos, como bocetos elaborados por el estudiante, textos escritos o esquemas. Los ocho estudiantes entrevistados se incluyeron en los dos primeros niveles del análisis, mientras que, para el tercer nivel, se incluyeron sólo aquellos con los que se hizo un seguimiento posterior.

\section{Discusión}

\section{La visualización como práctica académica en diseño}

Al indagar sobre el papel de la visualización en los primeros semestres de la formación se encontró que, aunque la bocetación es un recurso utilizado a menudo en los talleres de diseño y los docentes tratan de promover su práctica, el uso de métodos como la realización de esquemas y mapas conceptuales parece ocupar un lugar marginal. Para acercarse a la teoría se suele leer y elaborar informes sobre lo leído o hacer discusiones, pero, aunque la literatura señala las ventajas cognitivas que puede tener su utilización en el aprendizaje, la realización de esquemas es una herramienta poco utilizada. Al llegar a los últimos semestres, la realización de mapas conceptuales o esquemas se hace más común. En el desarrollo de los proyectos de grado, algunas de las personas entrevistadas, sin que se le preguntara específicamente al respecto, describieron la elaboración de gráficos para organizar las ideas o para optimizar la ejecución del proyecto. Se utilizan desde recursos análogos, como pegar papeles de colores en un tablero, hasta recursos digitales que implican el uso de algún programa vectorial para la construcción del diagrama, o recursos en línea creados especialmente para la elaboración de mapas conceptuales. Un ejemplo que utiliza rectángulos de papel para construir mapas conceptuales se encontró en la oficina de Miguel Bohórquez, docente del departamento (ver imagen 7). 


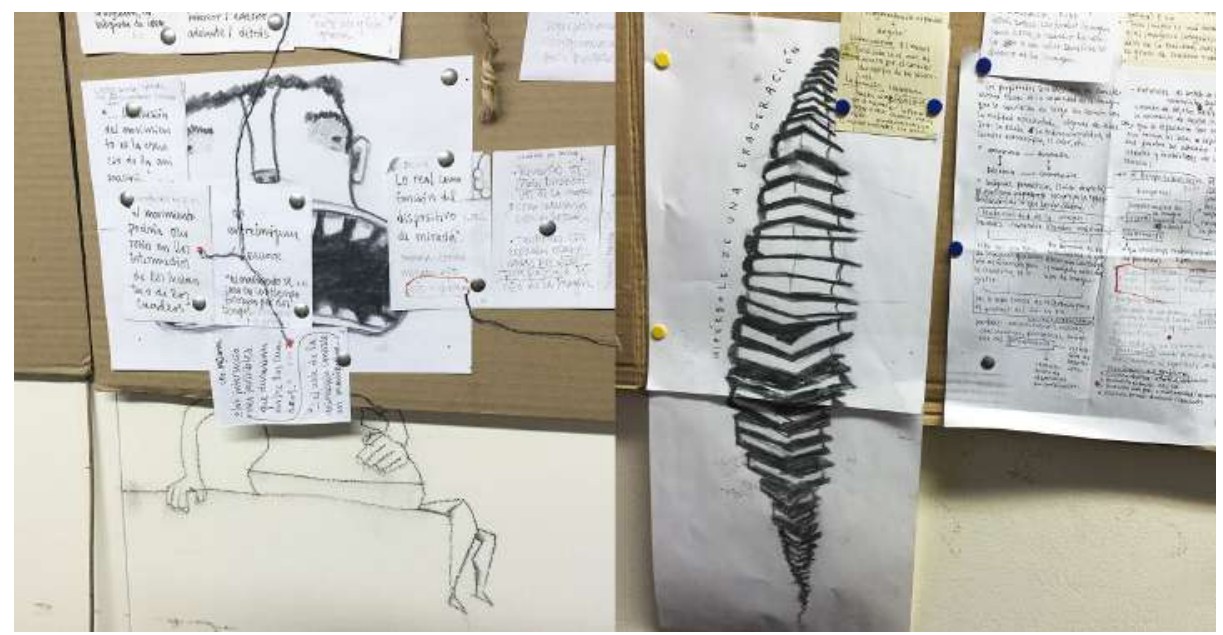

Imagen 7. Detalles de una pared en la oficina de Miguel Bohórquez con los hallazgos de su tesis de Doctorado en proceso. Nota: las conexiones se realizan con líneas dibujadas y en ocasiones con cuerdas que puede extender de una pared a otra buscando evidenciar la relación entre los conceptos.

Fuente: fotografías tomadas por los autores.

En las asignaturas de proyectos de grado 1 y 2 (como sugieren las entrevistas), algunos directores aconsejan a los estudiantes realizar un mapa conceptual como medio para revisar conceptos y aclarar vías de desarrollo del proyecto. En estos gráficos aparecen frases, símbolos e imágenes. Las imágenes tienen un propósito simbólico o representan de forma visual un concepto que es difícil de verbalizar; en otros casos la imagen aparece por otros motivos que evaden a razones prácticas, para inspirar, divertir o controvertir el texto. Por supuesto, a medida que avanza el proyecto, también aparecen bocetos de las alternativas de diseño. Estas propuestas no solo son una manera de materializar las decisiones de diseño, en ocasiones tienen un propósito investigativo en sí mismo al guiar intuitivamente la exploración gráfica e inclusive permiten al estudiante replantear el documento que evidencia el proceso de investigación. 
La visualización aparece entonces, en los últimos semestres de estudio, de distintas maneras: por un lado, como apoyo didáctico para organizar las ideas que guían la investigación, como es el caso de la realización de mapas conceptuales y esquemas, y, por otra parte, en la exploración gráfica que determina las alternativas de diseño, exploración que va desde la realización de los primeros bocetos hasta la elaboración de las propuestas finales.

En las entrevistas realizadas a los estudiantes y profesores de diseño, se encontró como una constante la valoración del proceso de diseño. La referencia a la bitácora de dibujo como una herramienta indispensable surgió en algunas ocasiones en las entrevistas y, en otras, el estudiante la llevaba mientras se realizaba la conversación, así no mencionara su uso durante la charla. Las bitácoras de proceso son muchas veces guardadas por el diseñador en formación como una manera de mantener el hilo en el desarrollo de ideas o proyectos. Por lo general de hojas blancas, contienen bocetos del avance del proyecto, junto a frases y esquemas. En las páginas aparecen tanto dibujos, donde se comienza a dar forma al proyecto de diseño, como algunos gráficos que ayudan al estudiante a pensar sobre el proyecto, e inclusive a comunicar esas reflexiones a colegas y profesores. Aunque la valoración de la importancia de dibujos y bocetos parece ser una constante, el lenguaje verbal mantiene un papel fundamental en el acercamiento conceptual al proyecto de diseño (imagen 8). Para algunos de los entrevistados, el proceso no necesariamente parte de la bocetación, para ellos, el punto de partida para reflexionar sobre las posibles soluciones a un proyecto son las palabras. Escribir algunos conceptos clave que guíen la investigación es un punto de partida que ayuda a la definición del proyecto al brindar un marco conceptual a la investigación. 


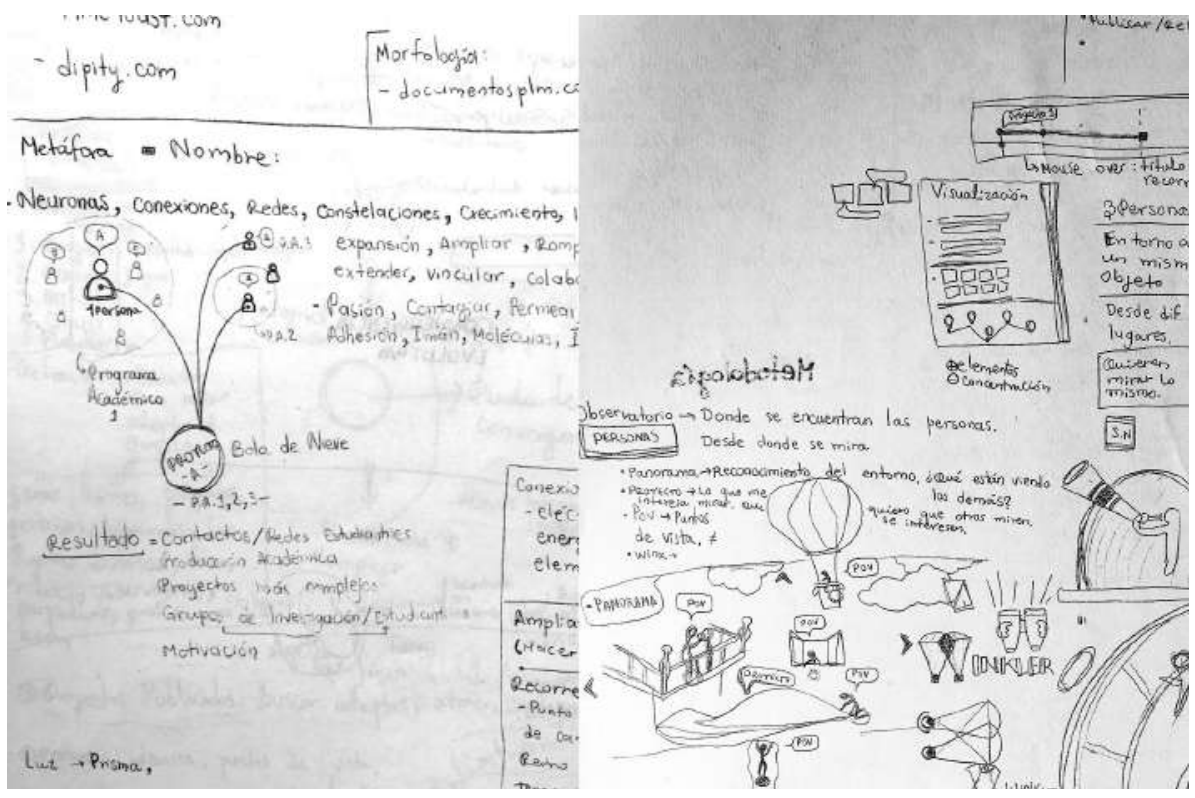

Imagen 8. Páginas de muestra del proceso de conceptualización del proyecto que se convertiría en el tema del trabajo de grado de Viviana Varón (fragmentos) Fuente: Viviana Varón, estudiante que participó en la investigación.

Al comparar el discurso del estudiante y el gráfico realizado se encontraron algunos datos interesantes. Al escuchar la descripción que los estudiantes daban de su trabajo y compararla con los gráficos que elaboraron en la primera entrevista, era evidente cómo la sustentación verbal por lo general era más rígida y estructurada, mientras en el gráfico emergían con mayor naturalidad dudas, ideas en formación y cambios de sentido.

Ocurrió en algunas ocasiones que el estudiante notaba durante la entrevista esa diferencia y hacía alguna anotación al respecto: 
¿Sabes que también he descubierto eso? Que cuando hablo en serio no puedo hablar sin dibujarlo (...) como que tengo que hacer al menos un círculo para hablar de lo que estoy hablando o al menos dibujarlo en el aire. No sé por qué (...) como que necesito ver lo que estoy diciendo (...). (A. Gardeazábal, comunicación personal, 2 de marzo de 2015)

En los estudiantes con los que se realizó un seguimiento posterior se hicieron evidentes cambios que se dieron en los proyectos de investigación y cómo estos cambios transformaron el discurso; se recopilaron además gráficos o bocetos de sus libros de proceso que ayudaron a tener una idea más clara sobre el avance de la investigación y cómo se transformaban los esquemas a medida que comenzaban a emerger nuevas inquietudes.

\section{Teoría y práctica en la investigación en Diseño}

La investigación académica, que está ligada en gran medida a la teorización y, por ende, a la lectura y escritura de textos, parece tener un choque simbólico con la naturaleza productiva de la práctica en diseño. Como se aclaraba en la introducción, los problemas con la escritura no son únicos de la carrera en diseño, pero el énfasis de la formación, que tiene como eje otro tipo de habilidades distintas a la escritura, acrecienta las dificultades. Al indagar sobre este problema en la investigación, se encontró que en general, los estudiantes reconocen la importancia de la lectura y la escritura en la formación, pero no relatan dentro de la carrera haber tenido mucha guía con respecto a cómo se escribe un texto académico.

El corte de la investigación, de tipo exploratorio, no permite generalizar si lo que reflejan las entrevistas es una tendencia, pero los testimonios recogidos son consecuentes con la literatura consultada que señala cómo por conceptos erróneos sobre los procesos de lectura y escritura se asume que el estudiante universitario ya debe dominar esas competencias y que la responsabilidad recae sobre otros niveles de escolaridad (Carlino, 2004). 
Sin embargo, si cada disciplina tiene sus códigos específicos y una particular manera de enmarcar el conocimiento que la diferencia de otras disciplinas, es necesario garantizar la interacción de los estudiantes con los géneros textuales y las formas discursivas que son propios de la comunidad académica en la que están ingresando. Se requiere, entonces, como lo señala Carlino "(...) la necesaria bienvenida a las culturas académicas":

Cada disciplina está hecha de prácticas discursivas propias, involucradas en su sistema conceptual y metodológico; aprender una materia no consiste solo en adquirir sus nociones y métodos sino manejar sus modos de leer y escribir característicos. [...] Ingresar a una comunidad disciplinaria implica compartir formas de interpretación y producción textual empleados en su dominio de conocimiento. (Carlino, 2004, p. 22)

La relación entre teoría y práctica es una discusión común entre diseñadores y estudiantes de diseño. El diseño es una carrera proyectual que, más que interesarse por construir teorías, busca aportar soluciones a un problema determinado. Al respecto dice Norberto Chaves: "la actividad específica del diseño gráfico es de naturaleza productiva, operativa, técnica, no se trata de un arte, una ciencia ni una tecnología científica sino de una práctica experimental con un alto componente artesanal y experimental, exploratorio o de ensayo y error" (Chaves, 2001, p. 139). Sin embargo, aunque el quehacer del diseño pueda aprenderse en la práctica, a las instituciones universitarias les compete una labor mucho más compleja, deben enseñar el conocimiento del contexto, de las relaciones sociales y culturales que dan sentido a la práctica (Chaves, 2001). Esta tensión que existe entre el pensamiento proyectual, que tiene una forma específica de entender la realidad para proponer cambios, y la teoría, que más que intervenir en los acontecimientos busca comprenderlos, aparece en las entrevistas como una dicotomía entre el escribir y el hacer, entre teorizar y diseñar; y lamentablemente esta oposición no logra resolverse. 
La falta de integración que existe entre ambos enfoques hace que el estudiante sienta que la revisión teórica y el proceso de escritura del documento es un protocolo formal que no necesariamente tiene relación con la propuesta de diseño. En algunos casos, inclusive, se hace la propuesta y luego se trata de estructurar el documento escrito de forma que justifique el resultado; el planteamiento teórico se considera como una imposición académica más que una herramienta indispensable para cumplir los objetivos de investigación.

En las entrevistas a estudiantes se preguntó cuál era para ellos la manera en que se articulaban las prácticas de lectura y escritura en el currículo. Apareció como una constante la opinión de que se lee más de lo que se escribe y que cuando se hacen trabajos escritos durante la formación no existe por lo general una retroalimentación de qué se hizo bien o mal en el texto, más allá de una nota numérica.

Sustentar la importancia de la visualización en la investigación en diseño no busca desdeñar la importancia de la escritura académica. La información recogida confirma la necesidad de implementar, desde la dirección del departamento de diseño, los procesos de escritura como una práctica unida al currículo. Así como los procesos de visualización pueden ser una herramienta para ayudar a definir variables en la investigación y apoyar de forma intuitiva al estudiante en su trabajo de grado, la escritura debe apoyar los proyectos prácticos para permitirle al estudiante reflexionar sobre el proceso de diseño y brindarle las herramientas necesarias para comunicar el alcance de su proyecto.

Aunque no se pretende que las prácticas de visualización reemplacen de ningún modo los procesos de escritura, las particularidades propias de la disciplina sugieren que es importante considerar cómo la visualización puede tener un lugar propio en la construcción del documento teórico para establecer un complemento que dé cuenta de esa parte del proceso que no 
necesariamente puede ser explicada a partir del lenguaje escrito. En este sentido la realización de infografías o mapas conceptuales puede ser un recurso no solo para guiar el proceso sino una manera de involucrar la visualización en la sustentación teórica. La reflexión teórica no debería verse como contraria a la experimentación práctica, es urgente buscar maneras de entender ambos elementos como fichas clave en la producción creativa.

\section{Conclusiones}

En el acompañamiento a los proyectos de grado de los estudiantes de últimos semestres de Diseño Gráfico se hicieron evidentes algunos conflictos con el proceso de investigación formativa. En general —salvo algunas excepcioneslos estudiantes consideran la investigación académica como un requisito desconectado de la práctica del diseño, y la elaboración del documento escrito se percibe como una tarea que interfiere con el objetivo final: el desarrollo del proyecto.

Existe una separación conceptual en el discurso de los estudiantes entre la teoría y la práctica, la investigación académica y el desarrollo del proyecto de diseño. Se considera al investigador como un teórico alejado de la disciplina del diseño, lo que confirma la necesidad de ajustar el proceso académico para integrar la práctica del diseño y la reflexión sobre esta práctica en una relación más armónica. Esta dicotomía refleja inquietudes registradas por algunos autores en la revisión de la literatura (Frayling,1993; Pontis 2009, Bonsiepe 2007).

Respecto a las prácticas de visualización en el proceso académico, la investigación confirma la importancia del pensamiento visual en el aprendizaje del diseño. La representación visual aparece en los procesos educativos de diferentes maneras. En los primeros semestres, durante el desarrollo de los 
proyectos, se recurre a bocetos y dibujos para explorar alternativas que ayuden a definir la dirección creativa de las propuestas gráficas. Durante los últimos semestres de la carrera se mantiene la utilización de estos recursos de representación y se hacen comunes otros recursos comunicativos poco usados anteriormente, como esquemas y diagramas conceptuales. Los esquemas permiten al estudiante estructurar conceptualmente el proyecto de investigación, entender sus motivaciones, y son un recurso comunicativo eficaz para explicar a pares y a profesores el avance del proyecto.

La incorporación consciente de métodos visuales dentro de las herramientas dadas a los estudiantes en las clases de metodología de investigación podría ayudar a que sean empleados con mayor eficacia recursos cognitivos adquiridos en sus años de formación, e inclusive colaborar a reducir la brecha percibida entre el ejercicio del diseño y la reflexión teórica sobre la práctica, requisito indispensable en el desarrollo de los proyectos de investigación. Futuras investigaciones podrían centrarse en la aplicación de estos recursos didácticos en las asignaturas relacionadas con la investigación formativa para comprobar si es posible definir una caja de herramientas metodológicas afín a los intereses particulares de la disciplina de diseño.

\section{Referencias}

Ausubel, D. P., Novak, J. y Hanesian, H. (1978). Educational Psychology. A Cognitive View (2a Ed.). New York: Holt, Rinehart And Winston.

Bonsiepe G. (2007). The Uneasy Relationship between Design and Design Research. En R. Michel (Ed.), Design Research Now. Board of International Research in Design (pp 25-39). Birkhäuser Basel. https://doi.org/10.1007/978-3-7643-8472-2_2 
Carlino, P. (2004). Escribir a través del currículum. Tres modelos para hacerlo en la universidad. Lectura y vida, 25(1), 16-27. https://media.utp.edu.co/ referencias-bibliograficas/uploads/referencias/articulo/248-escribir-a-travsdel-curriculum-tres-modelos-para-hacerlo-en-la-universidadpdf-jQyDBarticulo.pdf

Chaves, N. (2001). El oficio de diseñar. Propuestas a la consciencia crítica de los que comienzan. Gustavo Gili.

Cross, N. (2007) From a Design Science to a Design Discipline: Understanding Designerly Ways of Knowing and Thinking. En R. Michel (Ed.), Design Research Now. Board of International Research in Design (pp. 41-54). Birkhäuser Basel. https://doi.org/10.1007/978-3-7643-8472-2_3

Diezmann, C. (2009). The visual side to numeracy: students' sensemaking with graphics. Australian Primary Mathematics Classroom, 14(1), 16-20. https://www.researchgate.net/publication/27476219_The_visual_side_to_ numeracy_Students'_sensemaking_with_graphics

Findeli, A., Brouillet, D., Martin, S., Moineau, C. y Tarrago, R. (2008). Research Through Design and Transdisciplinarity: A Tentative Contribution to the Methodology of Design Research. En Swiss Design Network (Ed.), Focused Current Design Research Projects and Methods (pp. 67-88). Swiss Design Network Simpossium. https://www.academia.edu/14939794/Research_ through_design_and_transdisciplinarity_A_tentative_contribution_to_the_ methodology_of_design_research

Frayling, C. (1993). Research into Art \& Design. Royal College of Art. https://researchonline.rca.ac.uk/384/3/frayling_research_in_art_and_ design_1993.pdf

Friendly, M. (2008). Handbook of Computational Statistics: Data Visualization. Springer Berlin Heidelberg. 
Gardner, H. (1993). Estructuras de la mente. La teoría de las inteligencias multiples. Basic Books.

Hansen, Y. (2000). Visualization for thinking, planning, and problem solving. En R. Jacobson (Ed.), Information Design (pp. 193-220). The MIT Press.

Harley, J. (1987). The Map and the Development of the History of Cartography (Vol. I). The University of Chicago Press.

Heilman, K. (2005). Knowledge and talents. En K. Heilman, Creativity and the brain (pp. 27-58). Psychology Press.

Maya, J, y Mazo, E. P. (2020). Propiedades de las representaciones en diseño: una exploración interdisciplinaria de su rol funcional. Kepes, 17(21), 17-60. doi: 10.17151/kepes.2020.17.21.2

Moles, A. y Costa, J. (1991). Imagen didáctica. Ediciones Ceac.

Pontis, S. (2009). Diseño gráfico: un novel objeto de investigación. Caso de estudio: el proceso de diseño. Iconofacto, 5(6), 9-18. https://dialnet. unirioja.es/servlet/articulo?codigo $=5204254$

Rajamanickam, V. (2005). Infographics Seminar Handout. Industrial Design Center Indian Institute of Technology. http://www.schrockguide.net/ uploads/3/9/2/2/392267/infographic_handout.pdf

Restrepo, J. (2008). La investigación en los programas de diseño gráfico en Colombia. Iconofacto, 4(5), 114-121. https://dialnet.unirioja.es/servlet/ articulo?codigo $=5204290$

Rincón, G. y Gil, J. S. (2013). Lectura y escritura académica en la Universidad del Valle. Caracterización de prácticas y tendencias. Programa Editorial Universidad del Valle. 
Scrivener, S. (2009). The Roles of Art and Design Process and Object in Research. In N. Nimkulrat \& T. O'Riley (Eds.), Reflections and Connections. On the relationship between creative production and academic research. University of Art and Design Helsinki.

Self, J., Lee, S., y Bang, H. (2015). Perceptions of complexity in design representation: Implications for an understanding of design practice. The International Journal of Design Management and Professional Practice, 9(4), 33-46. https://www.researchgate.net/publication/298708197_ Perceptions_of_Complexity_in_Design_Representation_Implications_for_ an_Understanding_of_Design_Practice

Tufte, E. (1992). Envisioning Information. Graphics Press.

Tversky, B. (2005). Visuospatial Reasoning. En K. Holyoak, y R. Morrison (Eds.). The Cambridge Handbook of Thinking and Reasoning (pp. 209-241). Cambridge University Press.

Universidad del Valle. (2017). Reglamento de proyecto de grado. Diseño Industrial/Diseño Gráfico. Comité de programas académicos, Departamento de Diseño.

Como citar: Ramírez-Escobar, M. F. (2020). Prácticas de visualización en la investigación académica en diseño gráfico. Revista KEPES, 17(22), 77-108. https://doi.org/10.17151/kepes.2020.17.22.4 